\title{
Chave de identificação para caprelídeos (Crustacea, Amphipoda) do litoral dos Estados do Paraná e de Santa Catarina
}

\author{
Mariana Baptista Lacerda ${ }^{1,2}$ \& Setuko Masunari ${ }^{1}$ \\ ${ }^{1}$ Programa de Pós-graduação em Zoologia, Departamento de Zoologia, \\ Universidade Federal do Paraná - UFPR, Centro Politécnico, Bairro Jardim das Américas, CP 19020, \\ CEP 81531-980, Curitiba, PR, Brasil \\ ${ }^{2}$ Autor para correspondência: Mariana Baptista Lacerda, e-mail: lacerdamariana@yahoo.com.br
}

LACERDA, M.B. \& MASUNARI, S. Identification key for caprellids (Crustacea, Amphipoda) from coast of Paraná and Santa Catarina states. Biota Neotrop. 11(3): http://www.biotaneotropica.org.br/v11n3/ en/abstract?identification-key+bn00811032011

\begin{abstract}
An identification key was produced for caprellid species recorded from the coast of Paraná and Santa Catarina states, Southern Brazil. Analyzed specimens are part of scientific collections or were obtained from nature, within these states. Nine caprellid belonging to the family Caprellidae, from which eight of Caprellinae - Caprella danilevskii, Caprella dilatata, Caprella equilibra, Caprella penantis, Caprella scaura, Mayerella sp., Paracaprella pusilla e Pseudaeginella montoucheti - and one Phtisicinae - Phtisica marina - were treated in the present study.

Keywords: identification key, Caprellidae, Caprellinae, Phtisicinae, southern of Brazil.
\end{abstract}

LACERDA, M.B. \& MASUNARI, S. Chave de identificação para caprelídeos (Crustacea, Amphipoda) ocorrentes no litoral dos Estados do Paraná e de Santa Catarina. Biota Neotrop. 11(3): http://www. biotaneotropica.org.br/v11n3/pt/abstract?identification-key+bn00811032011

Resumo: Uma chave de identificação foi elaborada para as espécies de caprelídeos registradas no litoral dos estados do Paraná e de Santa Catarina. Os exemplares examinados fazem parte do acervo de coleções científicas ou são provenientes de coletas realizadas nestes estados. Nove espécies pertencentes à família Caprellidae, das quais oito Caprellinae - Caprella danilevskii, Caprella dilatata, Caprella equilibra, Caprella penantis, Caprella scaura, Mayerella sp., Paracaprella pusilla e Pseudaeginella montoucheti - e uma Phtisicinae - Phtisica marina - foram tratadas no presente estudo.

Palavras-chave: chave de identificação, Caprellidae, Caprellinae, Phtisicinae, sul do Brasil. 


\section{Introdução}

Caprelídeos são anfípodos marinhos caracterizados por possuírem abdômen reduzido, cabeça parcialmente fusionada com o primeiro segmento torácico e tendência a redução ou perda do terceiro e quarto par de pereópodos. Os pereópodos aparecem em número variado nas diferentes espécies, podendo possuir os cinco pares (pereópodos de 3-7) ou menos, como no caso do gênero Caprella, no qual os pares 3 e 4 são ausentes (Caine 1974).

Caracteristicamente, os últimos pares de pereópodos dos caprelídeos são utilizados para se prenderem aos substratos, mas, com o auxílio dos gnatópodos, se prestam também para locomoção. Os pleópodos utilizados nos demais grupos de anfípodos para natação são ausentes nos caprelídeos; entretanto, estes possuem capacidade de nadar, embora de modo limitado (Caine 1978).

De uma maneira geral, a taxonomia dos caprelídeos pode ser considerada complexa devido às variações morfológicas intraespecíficas em função da idade e do sexo (Guerra-García et al. 2006).

São conhecidas para o Brasil 19 espécies de caprelídeos divididas nas subfamílias Caprellinae e Phtisicinae, segundo revisão recente realizada por Myers \& Lowry (2003). Pertencem à Caprellinae 17 espécies: Caprella aculeata (Dana, 1853), C. andreae Mayer, 1890, C. danilevskii Czerniavski, 1868; C. dilatata Krøyer, 1843, C. equilibra Say, 1818, C. globiceps Dana, 1853, C. penantis Leach, 1814, C. scaura Templeton, 1836, Hemiaegina minuta Mayer, 1890, Liropus nelsonae Guerra-García, 2003, Monoliropus enodis Rayol \& Serejo, 2003, Orthoprotella melloi Quitete, 1975, Paracaprella digitimanus Quitete, 1971, P. pusilla Mayer, 1890, P. tenuis Mayer, 1903, Parvipalpus colemani Guerra-García, 2003 e Pseudaeginella montoucheti (Quitete, 1971). Duas espécies pertencem a Phtisicinae: Phtisica marina Slabber, 1769 e P. verae Quitete, 1979.

Para o Paraná, foram registradas C. danilevskii, C. penantis (Dubiaski-Silva \& Masunari 1995, Dutra 1988), C. equilibra, C. scaura e P. montoucheti (Neves 2006), enquanto para Santa Catarina, C. danilevskii, C. equilibra, C. penantis, C. scaura e P. pusilla (Dubiaski-Silva \& Masunari 2008).

Animais marinhos freqüentemente usam outros organismos como substrato, especialmente os sésseis como algas, esponjas, colônias de hidrozoários e briozoários, mas também os vágeis, como estrelas-do-mar (Volbehr \& Rachor 1997). Os caprelídeos são importantes representantes dessa fauna epibionte e se fixam no substrato envolvendo seus últimos pares de pereópodos nos ramos de algas, briozoários e hidrozoários (Thiel et al. 2003).

As espécies de caprelídeos são distribuídas mundialmente e várias delas são consideradas cosmopolitas; este fato provavelmente se deve às associações dos caprelídeos com substratos que apresentam alto potencial de flutuação, já que estes animais não apresentam estágio larval planctônico (fêmeas mantém os ovos em marsúpios, onde ocorre o desenvolvimento), e os adultos têm baixa capacidade de natação (McCain 1968, Thiel et al. 2003)

Os caprelídeos desempenham um importante papel nas cadeias tróficas nos ecossistemas marinhos como produtores secundários e terciários (Guerra-García 2004), atuando tanto como consumidores, como servindo de presas (Dauby et al. 2003). Participam fundamentalmente na dieta de muitos peixes (Caine 1987, 1989, 1991, Dubiaski-Silva \& Masunari 2008), de alguns moluscos cefalópodos nos primeiros estágios de vida (Pinczon du Sel et al. 2000) e de braquiúros (Dubiaski-Silva \& Masunari 2008).

Apesar da abundância e importância nas comunidades marinhas, os caprelídeos brasileiros têm sido pouco estudados, principalmente na região Sul do Brasil. Na tentativa de preencher esta lacuna, o presente trabalho tem como objetivo auxiliar e facilitar a identificação dos caprelídeos ocorrentes no litoral dos estados do Paraná e Santa Catarina, através do uso de uma chave pictórica de identificação.

\section{Material e Métodos}

Foram analisadas amostras de substratos biológicos (algas, colônias de ascídias, briozoários, cracas, hidrozoários, poliquetas e conchas de ostra e mexilhão), artificiais (cordas e bóias de cultivo de ostras e mexilhão) e sedimento contendo caprelídeos provenientes do litoral dos estados do Paraná e de Santa Catarina. Elas estão depositadas no Laboratório de Ecologia de Crustacea, pertencentes à Universidade Federal do Paraná, e no Museu de História Natural do Capão da Imbuia.

Além das amostras depositadas nas coleções científicas supracitadas, foram analisadas aquelas coletadas no mediolitoral inferior dos costões rochosos da Praia de Caiobá, Matinhos, PR, e de Bombinhas e Itapoá, SC, e na zona infralitoral do Arquipélago dos Tamboretes, Balneário Barra do Sul, SC e da Ilha do Xavier, Florianópolis, SC, em 2008 e 2009. Nestes últimos locais, as profundidades variaram de 1,5 a $11 \mathrm{~m}$ e as amostras foram obtidas com mergulho autônomo.

Os diferentes substratos biológicos foram isolados da rocha por raspagem com espátula, acondicionados em sacos plásticos e transportados em caixa térmica com gelo para posterior preservação do material em álcool etílico a $70 \%$ em laboratório. As amostras foram triadas sob microscópio estereoscópico e os indivíduos identificados com auxílio de bibliografia especializada. Os desenhos foram confeccionados com o auxílio de câmera clara acoplada a um microscópio óptico.

\section{Resultados e Discussão}

Foram encontradas nove espécies pertencentes à família Caprellidae, das quais oito da subfamília Caprellinae e uma da Phtisicinae. Apenas uma espécie foi identificada a nível de gênero devido à ausência de machos.

\section{Família Caprellidae Leach, 1814}

Subfamília Caprellinae Leach, 1814

\section{Caprella danilevskii Czerniavskii, 1868 (Figura 1a)}

Material examinado: Paraná: 17 espécimes em Sargassum; Santa Catarina: 1 espécime em Pterocladia, 25 espécimes em Sargassum.

Características de reconhecimento: corpo delgado e liso; cabeça sem espinhos ou projeções; peças bucais características do gênero (palpo mandibular ausente, molar presente, lobo externo do maxilípede igual ou maior que o lobo interno); própodo do gnatópodo 1 com dois espinhos preênseis proximais, margem do dáctilo serrada; dáctilo do gnatópodo 2 reduzido quando comparado com as demais espécies; brânquias elípticas com eixo longo usualmente paralelo ao corpo; ausência de espinhos preênseis no própodo dos pereópodos 5-7; abdômen nos machos com papila em forma de gancho na extremidade do apêndice.

Distribuição: Localidade tipo: Mar Negro (McCain \& Steinberg 1970).

Outros registros: França, Itália, Algeria, Africa do Sul, Japão, Austrália, Tasmânia (McCain 1968) e Brasil (Tabela 1).

Observações: Esta espécie tem ampla distribuição mundial e sua história de vida é uma das mais bem conhecidas dentre os Caprellidae (Díaz et al. 2005). Características que distinguem claramente esta espécie das demais encontradas na área são o abdômen diferenciado, dáctilo pequeno do gnatópodo 2 nos machos e ausência de espinhos preênseis nos pereópodos de ambos os sexos. Variações intraespecíficas no comprimento dos pereonitos 1 e 2 e da base do própodo do gnatópodo 2 atribuídas ao desenvolvimento foram 
Chave de identificação para os caprelídeos do litoral do Paraná e Santa Catarina

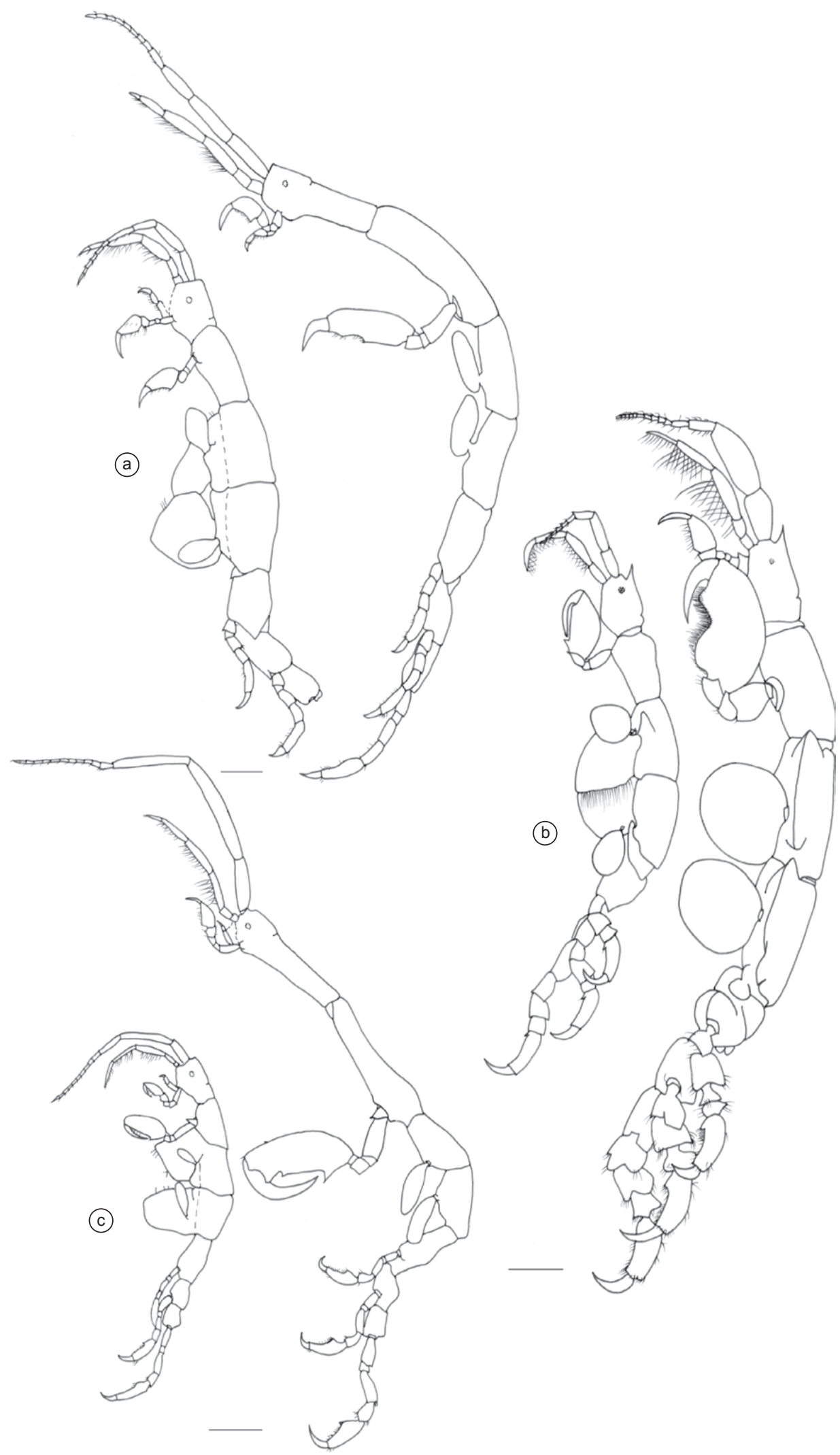

Figura 1. Machos (direita) e fêmeas (esquerda) em vista lateral. a) Caprella danilevskii (Redesenhado de Díaz et al. 2005), b) Caprella dilatata (Redesenhado de Masunari \& Takeuchi 2006), c) Caprella equilibra (Redesenhado de Díaz et al. 2005). Escalas: $1 \mathrm{~mm}$.

Figure 1. Males (rigth) and females (left) in lateral view. a) Caprella danilevskii (Redrawn from Díaz et al. 2005), b) Caprella dilatata (Redrawn from Masunari \& Takeuchi 2006), c) Caprella equilibra (Redrawn from Díaz et al. 2005). Scales: $1 \mathrm{~mm}$. 
registradas por Guerra-García \& Takeuchi (2004) para indivíduos coletados na Tasmânia, tendo os machos maiores as máximas dimensões, características estas observadas nos indivíduos analisados.

\section{Caprella dilatata Krøyer, 1843 (Figura 1b)}

Material examinado: Paraná: 25 espécimes em Pterocladia, 35 espécimes em Pterosiphonia, 13 espécimes em Sargassum; Santa Catarina: 15 espécimes em Bugula neritina, 48 espécimes em Didemnum perlucidum, 62 espécimes em cordas de cultivos de mexilhão, 26 espécimes em Pterocladia, 12 espécimes em Sargassum.
Características de reconhecimento: corpo robusto; cabeça com projeção direcionada anteriormente; peças bucais características do gênero; própodo do gnatópodo 1 com um par de espinhos preênseis, margem interna do própodo e do dáctilo serrada; palma do gnatópodo 2 proximalmente dilatada e convexa, com um espinho mediano, um processo trapezóide subdistalmente e muitas cerdas; brânquias arredondadas presentes nos pereonitos 3 e 4 , estes providos de pleuras auriculares bem desenvolvidas; palma do própodo dos pereópodos 5-7 ligeiramente côncavo com dois espinhos preênseis proximais,

Tabela 1. Registros das espécies de caprelídeos nas diversas localidades do Brasil. $\mathrm{X}^{*}$ : nova ocorrência das espécies em locais já estudados anteriormente.

Table 1. Records of caprellids species in different localities of Brazil. $X^{*}$ : new occurrence of species on places previously studied.

\begin{tabular}{|c|c|c|c|c|c|c|c|c|c|c|}
\hline Localidade & $\begin{array}{c}C . \\
\text { danilevskii }\end{array}$ & $\begin{array}{c}C . \\
\text { dilatata }\end{array}$ & $\begin{array}{c}C . \\
\text { equilibra }\end{array}$ & $\begin{array}{c}C . \\
\text { penantis }\end{array}$ & $\begin{array}{c}C . \\
\text { scaura }\end{array}$ & $\begin{array}{l}\text { Mayerella } \\
\text { sp. }\end{array}$ & $\begin{array}{c}P . \\
\text { pusilla }\end{array}$ & $\begin{array}{c}P . \\
\text { montoucheti }\end{array}$ & $\begin{array}{c}P . \\
\text { marina }\end{array}$ & Referência \\
\hline $\begin{array}{l}\text { Mar Grande e } \\
\text { Olivença, BA }\end{array}$ & - & - & - & - & - & - & - & $\mathrm{X}$ & - & Quitete 1971 \\
\hline Vitória, ES & - & - & - & - & - & - & - & $\mathrm{X}$ & - & Quitete 1971 \\
\hline Guarapari, ES & - & - & - & - & - & - & - & $\mathrm{X}$ & - & Quitete 1971 \\
\hline $\begin{array}{l}\text { Arraial do } \\
\text { Cabo, RJ }\end{array}$ & - & - & $\mathrm{X}$ & - & $\mathrm{X}$ & - & $\mathrm{X}$ & $\mathrm{X}$ & $\mathrm{X}$ & Serejo 1998 \\
\hline $\begin{array}{l}\text { Rio de } \\
\text { Janeiro, RJ }\end{array}$ & - & - & - & - & - & - & - & - & $\mathrm{X}$ & McCain 1968 \\
\hline Ubatuba, SP & - & - & - & - & $\mathrm{X}$ & - & $\mathrm{X}$ & - & - & Leite et al. 2007 \\
\hline Ubatuba SP & - & - & - & - & - & - & - & $\mathrm{X}$ & - & $\begin{array}{l}\text { Jacobucci } \\
\text { et al. } 2002\end{array}$ \\
\hline Ubatuba SP & $\mathrm{X}$ & $\mathrm{X}$ & $\mathrm{X}$ & - & $\mathrm{X}$ & - & - & $\mathrm{X}$ & - & $\begin{array}{l}\text { Jacobucci } \\
\text { et al. } 2009\end{array}$ \\
\hline Santos, SP & - & $\mathrm{X}$ & - & - & - & - & - & - & - & Jacobi 1987 \\
\hline Itanhaém, SP & $\mathrm{X}$ & - & - & - & - & - & - & - & - & $\begin{array}{l}\text { Tararam } \\
\text { et al. } 1986\end{array}$ \\
\hline Paranaguá, PR & $\mathrm{X}$ & - & - & $\mathrm{X}$ & - & - & - & - & - & Dutra 1988 \\
\hline Paranaguá, PR & - & - & $\mathrm{X}$ & - & $\mathrm{X}$ & - & - & $\mathrm{X}$ & - & Neves 2006 \\
\hline Matinhos, PR & $\mathrm{X}$ & - & - & $\mathrm{X}$ & - & - & - & - & - & $\begin{array}{c}\text { Dubiaski-Silva \& } \\
\text { Masunari } 1995\end{array}$ \\
\hline Penha, SC & - & $\mathrm{X}$ & - & - & - & - & - & - & - & $\begin{array}{c}\text { Masunari \& } \\
\text { Takeuchi } 2006\end{array}$ \\
\hline Bombinhas, SC & $\mathrm{X}$ & - & $\mathrm{X}$ & $X$ & $\mathrm{X}$ & - & $\mathrm{X}$ & - & - & $\begin{array}{c}\text { Dubiaski-Silva \& } \\
\text { Masunari } 2008\end{array}$ \\
\hline $\begin{array}{l}\text { Governador Celso } \\
\text { Ramos, SC }\end{array}$ & & - & - & $\mathrm{X}$ & - & - & - & - & - & $\begin{array}{l}\text { Mittmann \& } \\
\text { Müller } 1998\end{array}$ \\
\hline Paranaguá, PR & - & $\mathrm{X}^{*}$ & $X^{*}$ & - & - & $X^{*}$ & $\mathrm{X}^{*}$ & - & - & Presente estudo \\
\hline Guaratuba, PR & - & $\mathrm{X}$ & $\mathrm{X}$ & - & $\mathrm{X}$ & - & $\mathrm{X}$ & - & - & Presente estudo \\
\hline Matinhos, PR & $\mathrm{X}$ & $\mathrm{X}^{*}$ & $\mathrm{X}^{*}$ & $\mathrm{X}$ & - & - & $X^{*}$ & - & - & Presente estudo \\
\hline Ilha do Saí, SC & $\mathrm{X}$ & $\mathrm{X}$ & - & $\mathrm{X}$ & - & - & - & - & - & Presente estudo \\
\hline Itapoá, SC & $\mathrm{X}$ & $\mathrm{X}$ & - & - & - & - & $\mathrm{X}$ & - & - & Presente estudo \\
\hline Barra do Sul, SC & - & $\mathrm{X}$ & $\mathrm{X}$ & - & $\mathrm{X}$ & - & $\mathrm{X}$ & $\mathrm{X}$ & $\mathrm{X}$ & Presente estudo \\
\hline Penha, SC & - & $\mathrm{X}$ & $\mathrm{X}^{*}$ & - & $\mathrm{X}^{*}$ & - & $\mathrm{X}^{*}$ & - & - & Presente estudo \\
\hline Bombinhas, SC & $\mathrm{X}$ & $\mathrm{X}^{*}$ & $\mathrm{X}$ & - & $\mathrm{X}$ & - & - & - & - & Presente estudo \\
\hline Florianópolis, SC & - & - & - & - & - & - & - & - & $\mathrm{X}$ & Presente estudo \\
\hline
\end{tabular}




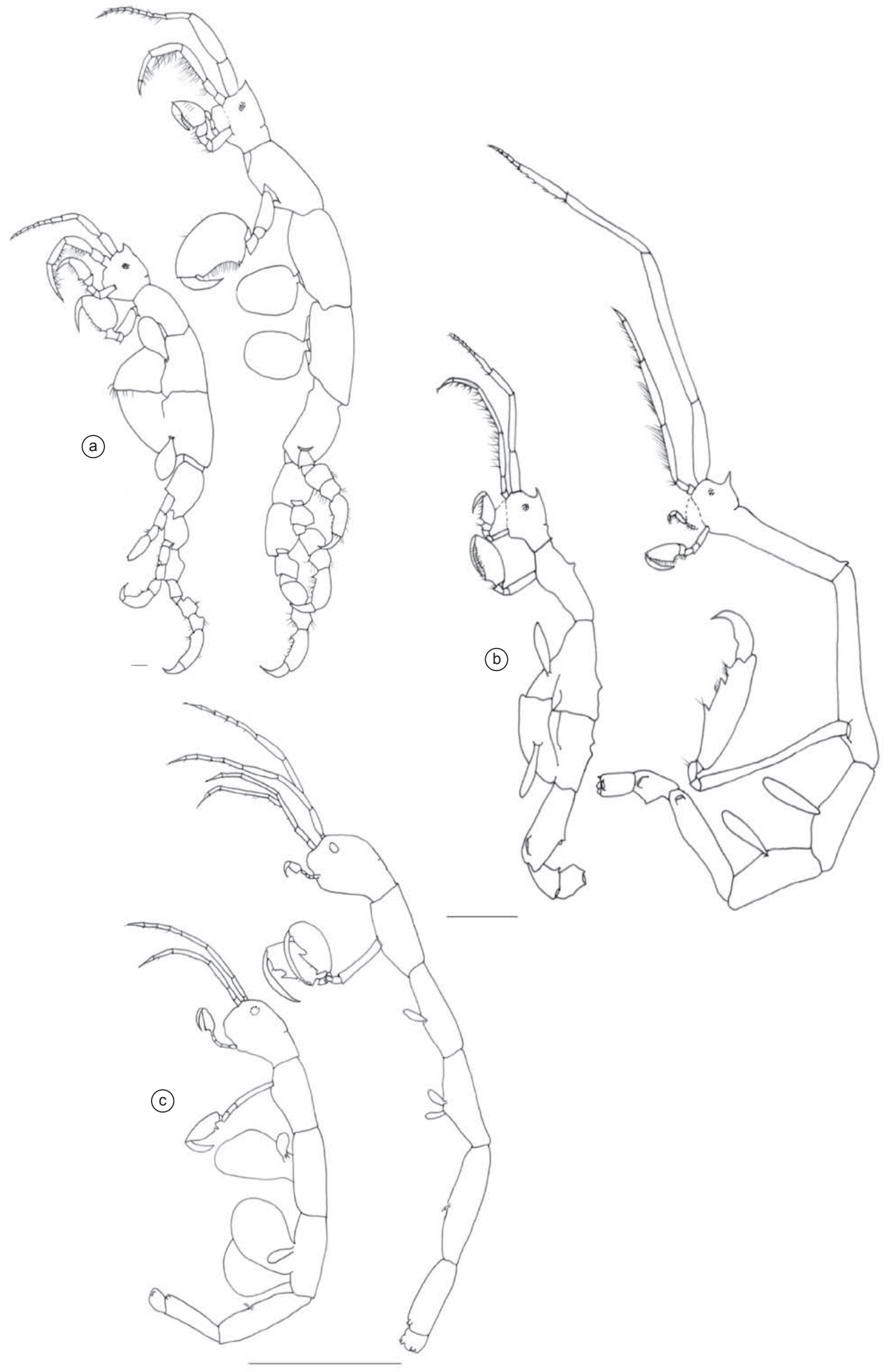

Figura 2. Machos (direita) e fêmeas (esquerda) em vista lateral. a) Caprella penantis (Redesenhado de Guerra-García et al. 2006), b) Caprella scaura. (Redesenhado de Guerra-García 2004), c) Mayerella sp. Escalas: $1 \mathrm{~mm}$.

Figure 2. Males (rigth) and females (left) in lateral view. a) Caprella penantis (Redrawn from Guerra-García et al. 2006), b) Caprella scaura. (Redrawn from Guerra-García 2004). c) Mayerella sp. Scales: $1 \mathrm{~mm}$. 
dáctilo curvado; abdômen nos machos com um par de apêndices biarticulados, um par de lobos medianos e um lobo dorsal único, nas fêmeas com um par de lobos laterais e um lobo dorsal com um par de cerdas plumosas; segundo artículo do pedúnculo da antena 1 dilatado nos machos.

Distribuição: Localidade tipo: Rio de Janeiro, Brasil (Krøyer 1843).

Outros registros: Espanha, França, Itália, Egito, Algeria e Ceuta (Krapp-Shickel 1993, Guerra-García \& Takeuchi 2002) e Brasil (Tabela 1).

Observações: Essa espécie é muito semelhante a $C$. penantis, podendo ser diferenciada pelo gnatópodo 2 com palma proximalmente dilatada e convexa com um espinho mediano e um processo trapezóide subdistalmente (Krapp-Schickel 1993), ausência de espinho proximal no própodo do gnatópodo 2 e pereonitos 3 e 4 providos de pleuras auriculares bem desenvolvidas. Segundo Guerra-García et al. (2006), além das características do gnatópodo 2, as espécies podem ser diferenciadas ainda por diferenças no tamanho do corpo e forma das brânquias (alongadas em C. penantis e redondas em C. dilatata). Entretanto, juvenis de $C$. dilatata possuem um espinho proximal no própodo do gnatópodo 2 , o que pode ser confundido com os adultos de C. penantis.

\section{Caprella equilibra Say, 1818 (Figura 1c)}

Material examinado: Paraná: 26 espécimes em bóias e cordas de cultivos de ostras, 2 espécimes em sedimento; Santa Catarina: 24 espécimes em Bugula neritina, 61 espécimes em cordas de cultivos de mexilhão, 4 espécimes em Didemnum perlucidum, 1 espécime em Sargassum.

Características de reconhecimento: corpo delgado e liso exceto pelo espinho entre as inserções dos gnatópodos 2, pereonitos 1-2 muito alongados nos machos; cabeça sem espinho ou projeções; peças bucais características do gênero; própodo do gnatópodo 1 com dois espinhos preênseis proximais, margem do própodo e do dáctilo serrada; base do gnatópodo 2 menor do que metade do comprimento do pereonito 2; brânquias elípticas; própodo dos pereópodos 5-7 robusto com dois espinhos preênseis proximais; abdômen do macho com um par de apêndices e um par de lobos e da fêmea com um par de lobos, típicos do gênero.

Distribuição: Localidade tipo: Carolina do Sul (Say 1818).

Outros registros: França, Itália, Iugoslávia, Turquia, Israel e Egito (Krapp-Schickel 1993), África do Sul, Madagascar, Estados Unidos, Argentina, Chile, Panamá, Japão, Austrália, Nova Zelândia, Tasmânia, Singapura, Malásia (McCain 1968) e Brasil (Tabela 1).

Observações: Os indivíduos dessa espécie apresentam uma projeção ventral distinta entre os gnatópodos 2 a qual pode ser utilizada para a diferenciação dessa espécie das demais encontradas na região. McCain (1968) encontrou variações nesse espinho ventral entre os gnatópodos 2, podendo os mesmos faltarem em algumas populações do Atlântico Norte. Porém, essa variação não foi observada nos indivíduos estudados, todos os quais possuindo o dito espinho ventral. As demais estruturas estão de acordo com o referido autor.

\section{Caprella penantis Leach, 1814 (Figura 2a)}

Material examinado: Paraná: 14 espécimes em Gymnogongrus, 52 espécimes em Pterocladia, 6 espécimes em Pterosiphonia, 6 espécimes em Sargassum, 5 espécimes em Sertularia marginata; Santa Catarina: 5 espécimes em Sargassum.

Características de reconhecimento: corpo robusto; cabeça com projeção direcionada anteriormente; peças bucais características do gênero; própodo do gnatópodo 1 com dois espinhos preênseis proximais, margem do própodo e dáctilo serrada; base do gnatópodo 2 menor que o pereonito 2, própodo com um espinho proximal; brânquias arredondadas; própodo dos pereópodos 5-7 com um par de espinhos preênseis proximais; abdômen dos machos e fêmeas típico do gênero.

Distribuição: Localidade tipo: Devonshire, Reino Unido (McCain \& Steinberg 1970).

Outros registros: África do Sul, Austrália, Estados Unidos, Espanha, França, Japão, Nova Zelândia e Portugal (McCain 1968) e Brasil (Tabela 1).

Observações: Esta espécie é amplamente distribuída e provavelmente se trata de um complexo de espécies em que é difícil de entender se a variação morfológica é intra ou interespecífica. Guerra-García et al. (2006) percebendo essa dificuldade sentiram a necessidade de encontrar uma ferramenta genética para resolver estes problemas taxonômicos. Dessa forma, usaram uma abordagem molecular preliminar (RAPD) para explorar essas variações intraespecíficas em populações de $C$. penantis do Estreito de Gibraltar, e concluíram que variações morfológicas entre populações de $C$. penantis provavelmente são influenciadas por características ecológicas dos habitats.

\section{Caprella scaura typica Mayer, 1890 (Figura 2b)}

Material examinado: Paraná: 27 espécimes em conchas de ostras em cultivos; Santa Catarina: 12 espécimes em Amphiroa beauvoissi, 3 espécimes em Didemnum perlucidum, 28 espécimes em Sargassum, 1 espécime em Spyridia aculeata.

Características de reconhecimento: corpo delgado, nos machos espinhos dorsais não proeminentes e nas fêmeas corpo geralmente espinhoso, pereonitos 1-2 longos e muito alongados nos machos; cabeça com um espinho direcionado anterodistalmente; peças bucais características do gênero; própodo do gnatópodo 1 com dois espinhos preênseis proximais, margem do própodo e dáctilo serrada; base do gnatópodo 2 aproximadamente do mesmo comprimento do pereonito 2, própodo alongado nos machos e palma com dois espinhos e uma projeção triangular distal; brânquias elípticas com eixo maior perpendicular ao comprimento do pereonito; própodo dos pereópodos 5-7 com dois espinhos preênseis proximais; abdômen dos machos e fêmeas típico do gênero.

Distribuição: Localidade tipo: Rio de Janeiro, Brasil (Krapp et al. 2006).

Outros registros para Caprela scaura s.l: cosmopolita (Krapp et al. 2006) e Brasil (Tabela 1).

Observações: Krapp et al. (2006) revisaram C. scaura com base no material do Mediterrâneo e diferenciam a espécie em grupos com espinhos ventrais e sem espinhos ventrais. Destas, citam 2 subespécies para o Brasil sem espinhos ventrais, C. scaura typica Mayer, $1890 \mathrm{e}$ C. scaura cornuta Mayer, 1890. Os indivíduos examinados no presente estudo apresentam morfologia mais próxima à primeira variedade, ou seja, machos com espinho curto, agudo e curvado para frente na cabeça; em adultos o flagelo da antena 1 com 8-9 artículos incompletamente definidos e 11 bem definidos; os pereonitos 1 a 3 dos machos maiores com um espinho dorsodistal e o pereonito 2 com um par dorsal na região da inserção dos gnatópodos 2. No entanto, foram observados espinhos também nos pereonitos 4 a 7 , sendo um dorsodistal no pereonito 4, dois pares dorsais no pereonito 5 e um par próximo da inserção dos pereópodos 5 , um par dorsal no pereonito 6 e um par próximo da inserção dos pereópodos 6 e um espinho dorsodistal no pereonito 7. Krapp et al. (2006) afirmam ainda que nesta subespécie o dáctilo do gnatópodo 2 não apresenta projeções na margem interna, porém, em alguns indivíduos esta projeção foi observada. As demais características como corpo com cerdas e fêmeas com o corpo variando de desprovido de espinhos a extremamente espinhoso estão de acordo. 


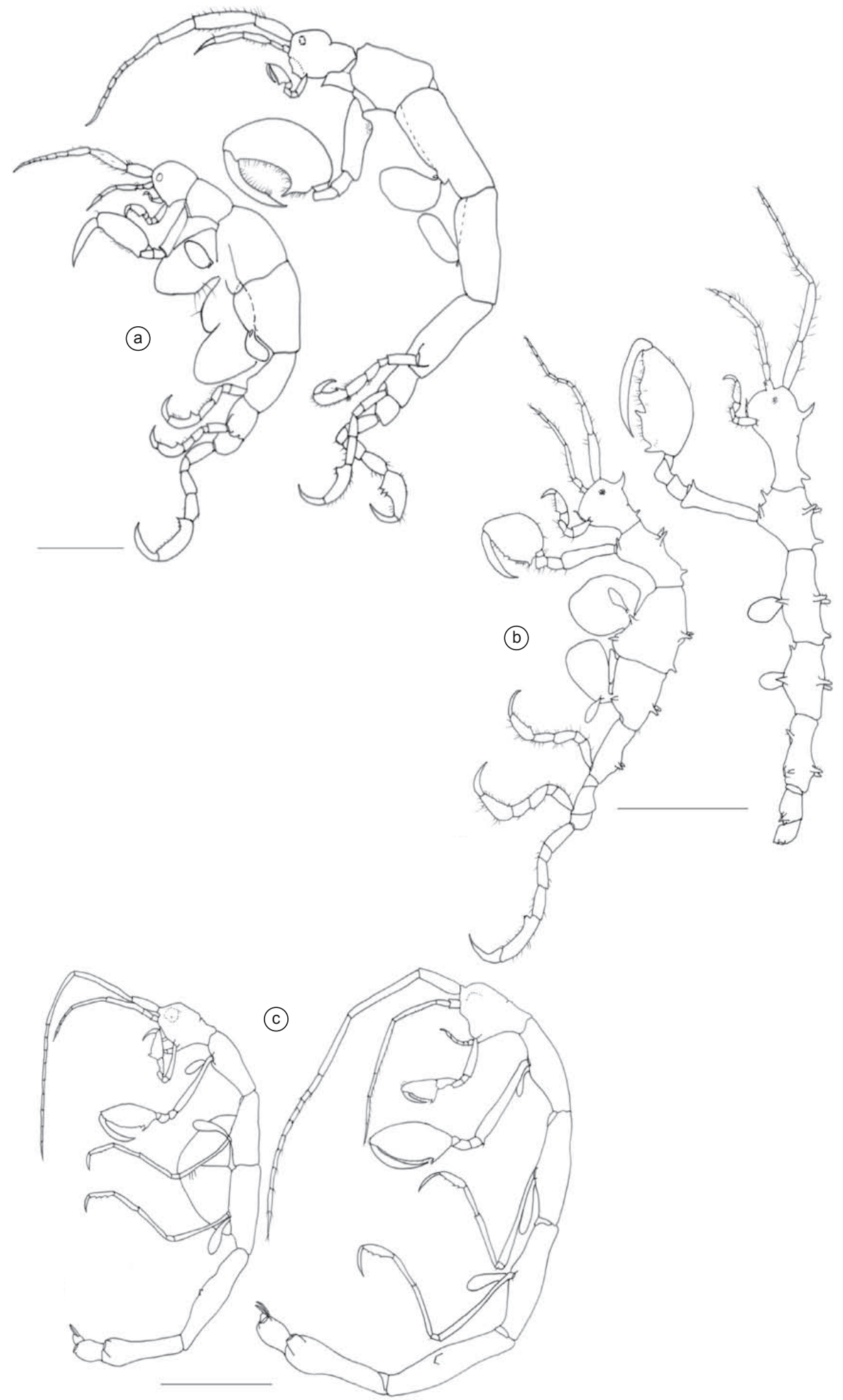

Figura 3. Machos (direita) e fêmeas (esquerda) em vista lateral. a) Paracaprella pusilla. (Redesenhado de Díaz et al. 2005), b) Pseudaeginella montoucheti, c) Phtisica marina (Redesenhado de McCain 1968). Escalas: $1 \mathrm{~mm}$.

Figure 3. Males (rigth) and females (left) in lateral view. a) Paracaprella pusilla. (Redrawn from Díaz et al. 2005), b) Pseudaeginella montoucheti, c) Phtisica marina (Redrawn from McCain 1968). Scales: $1 \mathrm{~mm}$. 


\section{Mayerella sp. (Figura 2c)}

Material examinado: Paraná: 3 espécimes em sedimento.

Características de reconhecimento: Os indivíduos foram identificados como gênero Mayerella baseando-se na combinação das seguintes características: antena 2 sem cerdas natatórias, flagelo biarticulado; palpo da mandíbula com três segmentos, uma única cerda no artículo terminal; molar presente; placa externa do maxilípede maior do que a interna; brânquias nos pereonitos 3 e 4; pereópodos 3 e 4 com dois segmentos, pereópodo 5 com três segmentos; abdômen do macho com um par de apêndices e um par de lobos, abdômen da fêmea sem par de apêndices, mas com lobos laterais (McCain 1968). No entanto, devido a poucos exemplares encontrados, sendo dois jovens e uma fêmea, não foi possível a identificação em nível específico.

\section{Paracaprella pusilla Mayer, 1890 (Figura 3a)}

Material examinado: Paraná: 28 espécimes em conchas de cultivo de ostras, 1 espécime em sedimento; Santa Catarina: 7 espécimes em Dynamena crisioides, 5 espécimes em cordas de cultivo de mexilhão, 2 espécimes em Phragmatopoma, 2 espécimes em Sertularia marginata.

Características de reconhecimento: corpo delgado e liso, machos com larga projeção triangular na margem anteroventral do pereonito 2 e pleuras desenvolvidas nos pereonitos 3 e 4; cabeça sem espinho ou projeções; palpo mandibular ausente, representado por uma única cerda, molar presente, lobo externo do maxilípede maior que o interno; própodo do gnatópodo 1 com um espinho preênsil proximal, margem do própodo e do dáctilo serrada; base do gnatópodo 2 pequena e expandida com protuberância proximal na margem posterior, própodo com entalhe superficial arredondado mediano e dáctilo com cerdas; brânquias ovais; pereópodos 3 e 4 presentes, com dois artículos minúsculos, sendo o terminal o menor e provido de 2 cerdas; abdômen dos machos com um par de apêndices e um par de lobos com cerdas, apêndices com cerda na base e na extremidade, o das fêmeas com um par de lobos com uma ou duas cerdas.

Distribuição: Localidade tipo: Rio de Janeiro, Brasil (McCain 1968).

Outros registros: Canal de Suez, China, Jamaica e Tanzânia, (McCain 1968) e Brasil (Tabela 1).

Observações: Variações na projeção triangular da margem anteroventral do pereonito 2, nas pleuras dos pereonitos 3 e 4 e na profundidade do entalhe do própodo do gnatópodo 2 foram observadas. Essas estruturas comumente são mais conspícuas nos machos maiores, no entanto, elas podem ser observadas mesmo de forma sutil nos machos menores.

\section{Pseudaeginella montoucheti (Quitete, 1971) (Figura 3b)}

Material examinado: Santa Catarina: 12 espécimes em Amphiroa beauvoissi, 1 espécime em Spyridia aculeata.

Características de reconhecimento: corpo espinhoso nos machos e fêmeas; cabeça com espinho dirigido anteriormente, pereonitos 1-6 providos com espinhos sendo, pereonito $1 \mathrm{com}$ um espinho dorsal posterior; pereonito 2 com um par de espinhos dorsais medialmente, um espinho dorsal posterior, um par de espinhos laterais anteriormente e um par de espinhos laterais medialmente; pereonito 3 com um par de espinhos dorsais medialmente, um espinho dorsal posterior e um par de espinhos laterais medialmente; pereonito 4 com um par de espinhos dorsais medialmente, um espinho dorsal posterior, um par de espinhos laterais anteriormente e um par de espinhos laterais medialmente; pereonito 5 com um par de espinhos dorsais medialmente, um par de espinhos laterais anteriormente, um par de espinhos laterais medialmente perto da inserção dos pereópodos 5; pereonito $6 \mathrm{com}$ um par de espinhos laterais medialmente perto da inserção dos pereópodos 6; palpo mandibular com três segmentos, segundo segmento com uma cerda simples e terceiro com uma protuberância e fórmula setal $1-6-1$; própodo do gnatópodo 1 subtriangular, palma com um par de espinhos preênseis e cerdas; própodo do gnatópodo 2 oval, margem interna com uma cerda proximal, três projeções triangulares medialmente e distalmente com algumas cerdas, comprimento do dáctilo menor do que o da palma com cerdas na margem interna; brânquias ovaladas; pereópodos 3 e 4 minúsculos, com um artículo, provido de duas cerdas simples apicalmente, pereópodos 5-7 com seis segmentos; abdômen dos machos com um par de lobos laterais e um lobo dorsal com um par de cerdas dorsais.

Distribuição: Localidade tipo: Pernambuco, Brasil (Quitete 1971).

Outros registros: Brasil (Tabela 1).

Observações: Pseudaeginella montoucheti foi descrita baseada em indivíduos coletados na região nordeste do Brasil e pertencente ao gênero Fallotritella. Sugestões de sinonímia entre Fallotritella e Pseudaeginella foram registradas por alguns autores (McCain 1968, Laubitz 1993), porém, devido à ausência de material e da localização do material tipo de Pseudaeginella para fins de comparação dos referidos gêneros, não foi possível consolidar a dita sinonímia. Mais tarde, Laubitz (1995) examinou indivíduos de Pseudaeginella tristanensis e levou em consideração as observações dos referidos autores, considerando válida a sinonímia, baseando-se principalmente na presença de pereópodos 3 e 4 minúsculos, com um artículo. A presente espécie poder ser facilmente diferenciada das demais pela presença de numerosos espinhos nos pereonitos.

Subfamília Phtisicinae Vassilenko, 1968

Phtisica marina Slabber, 1769 (Figura 3c)

Material examinado: Santa Catarina: 20 espécimes em Amphiroa beauvoissi.

Características de reconhecimento: corpo delgado e liso; cabeça sem espinho ou projeção; palpo mandibular com três segmentos, molar ausente, lobo externo do maxilípede igual ao lobo interno; própodo do gnatópodo 1 triangular com 4 ou 5 espinhos preênseis, margem do própodo e dáctilo não serrada; própodo do gnatópodo 2 com um espinho preênsil grande e um pequeno, palma com vários espinhos pequenos e cerdas; brânquias elípticas presentes na base dos pereonitos 2-4; pereópodos 3 e 4 bem desenvolvidos e com seis segmentos, palma do própodo com três espinhos pequenos, pereópodos 5 com cinco segmentos e sem espinhos, pereópodos 6 e 7 com seis segmentos com dois espinhos preênseis proximais, um espinho mediano e um distal; abdômen dos machos com quatro apêndices biarticulados e um par piriforme com uma cerda terminal; abdômen da fêmea com dois pares de apêndices biarticulados, par de lobos e uma projeção anterior.

Distribuição: Localidade tipo: Província de Zeeland, Holanda (Krapp-Schickel 1993).

Outros registros: amplamente distribuída nos oceanos Atlântico e Pacífico e no Mar Mediterrâneo (McCain 1968, Krapp-Schickel 1993). Brasil (Tabela 1).

Observações: McCain (1968) registrou a espécie para o Rio de Janeiro, porém, o presente estudo constitui a primeira ocorrência para Santa Catarina. Ela pode ser diferenciada facilmente das demais espécies encontradas na área de estudo pela presença de brânquias no pereonito 2, pereópodos 3 e 4 com seis segmentos e abdômen dos machos com quatro apêndices.

\section{Agradecimentos}

Aos responsáveis pelo Laboratório de Estudos de Cnidaria, Laboratório de Ecologia e Sistemática de Ascidiacea e Laboratório de Bentos do Centro de Estudos do Mar pertencentes à Universidade Federal do Paraná pela doação das amostras de caprelídeos, e ao Museu de História Natural do Capão da Imbuia pela disponibilização das amostras depositadas em suas coleções. Ao Conselho Nacional de Desenvolvimento Cientifico e Tecnológico (CNPq) pela bolsa de mestrado concedida à primeira autora. Esta é a contribuição 1832 do Departamento de Zoologia da Universidade Federal do Paraná. 


\section{Referências Bibliográficas}

CAINE, E.A. 1974. Comparative functional morphology of feeding in three species of caprellids (Crustacea, Amphipoda) from the northwestern Florida Gulf Coast. J. Exp. Mar. Biol. Ecol. 15:81-96. http://dx.doi. org/10.1016/0022-0981(74)90065-3

CAINE, E.A. 1978. Habitat adaptations of North American caprellid Amphipoda (Crustacea). Biol. Bull. 155:288-296. http://dx.doi. org/10.2307/1540953

CAINE, E.A. 1987. Potential effect of floating dock communities on a South Carolina estuary. J. Exp. Mar. Biol. Ecol. 108:83-91. http://dx.doi. org/10.1016/0022-0981(87)90132-8

CAINE, E.A. 1989. Caprellid amphipod behaviour and predatory strikes by fish. J. Exp. Mar. Biol. Ecol. 126:173-180. http://dx.doi.org/10.1016/00220981(89)90088-9

CAINE, E.A. 1991. Caprellid amphipods: fast food for the reproductively active. J. Exp. Mar. Biol. Ecol. 148:27-33. http://dx.doi.org/10.1016/00220981(91)90144-L

DAUBY, P., NYSSEN, F. \& DE BROYER, C. 2003. Amphipods as food sources for higher trophic levels in the Southern Ocean: a synthesis. In Antarctica in a Global Context (A. Huiskes, W.W.C. Gieskes, J. Rozema, R.M.L. Schorno, S.M. van der Vies \& W.J. Wolff, eds). Backhuys, Leiden, p.129-134.

DÍAZ, Y.J., GUERRA-GARCÍA, J.M. \& MARTÍN, A. 2005. Caprellids (Crustacea: Amphipoda: Caprellidae) from shallow waters of the Caribbean coast of Venezuela. Org. Divers. Evol. 5(10):1-25.

DUBIASKI-SILVA, J. \& MASUNARI, S. 1995. Ecologia populacional dos Amphipoda (Crustacea) dos fitais de Caiobá, Paraná. Rev. Bras. Zool. 12(2):373-396. http://dx.doi.org/10.1590/S0101-81751995000200015

DUBIASKI-SILVA, J. \& MASUNARI, S. 2008. Natural diet of fish and crabs associated with the phytal community of Sargassum cymosum C. Agardh, 1820 (Phaeophyta, Fucales) at Ponta das Garoupas, Bombinhas, Santa Catarina State, Brazil. J. Nat. Hist. 42(27-28):1907-1922. http://dx.doi. org/10.1080/00222930802126896

DUTRA, R.R.G. 1988. A fauna vágil do fital Pterocladia capillacea (Rhodophyta Gelidiaceae) Ilha do Mel, Paraná, Brasil. Rev. Bras. Biol. 48(3):589-605.

GUERRA-GARCIA, J.M. 2004. The Caprellidea (Crustacea, Amphipoda) from Western Australia and Northern Territory, Australia. Hydrobiologia 522:1-74. http://dx.doi.org/10.1023/B:HYDR.0000029929.07691.a7

GUERRA-GARCIA, J.M. \& TAKEUCHI, I. 2002. The Caprellidea (Crustacea: Amphipoda) from Ceuta, North Africa, with the description of three species of Caprella, a key to the species of Caprella, and biogeographical discussion. J. Nat. Hist. 36:675-713. http://dx.doi. org/10.1080/00222930010025923

GUERRA-GARCIA, J.M. \& TAKEUCHI, I. 2004. The Caprellidea (Crustacea: Amphipoda) from Tasmania. J. Nat. Hist. 38:967-1044. http:// dx.doi.org/10.1080/0022293021000054497

GUERRA-GARCÍA, J.M., REDONDO-GÓMEZ, S., ESPINA, Á., CASTILLO, J., LUQUE, T., GARCÍA-GÓMEZ, J.C. \& FIGUEROA, M.E. 2006. Caprella penantis Leach, 1814 and Caprella dilatata Kroyer, 1843 (Crustacea: Amphipoda) from the Strait of Gibraltar: a molecular approach to explore intra- and interspecific variation. Mar. Biol. Res. 2:100-108. http://dx.doi.org/10.1080/17451000600672511

JACOBI, C.M. 1987. Spatial and temporal distribution of Amphipoda associated with mussel beds from the Bay of Santos, Brazil. Mar. Ecol. Prog. Ser. 35:51-58. http://dx.doi.org/10.3354/meps035051

JACOBUCCI, G.B., MORETTI, D., SILVA, E.M. \& LEITE, F.P.P. 2002. Caprellid amphipods on Sargassum cymosum (Phaeophyta): depth distribution and population biology. Nauplius 10(1):27-36.
JACOBUCCI, G.B., TANAKA, M.O. \& LEITE, F.P.P. 2009. Temporal variation of amphipod assemblages associated with Sargassum filipendula (Phaeophyta) and its epiphytes in a subtropical shore. Aquat. Ecol. 43(4):1031-1040. http://dx.doi.org/10.1007/s10452-009-9230-2

KRAPP, T., LANG, C., LIBERTINI, Â., MELZER, R.R. 2006 Caprella scaura Templeton, 1836 sensu lato (Amphipoda: Caprellidae) in the Mediterranean. Org. Divers. Evol. 6, Electr. Suppl. 3:1-18.

KRAPP-SCHICKEL, T. 1993. Subordem Caprellidea. In The Amphipoda of the Mediterranean (S. Ruffo, ed.). Mémoires de L'Institute Oceanographique, Monaco, v. 13, n. 3, p.773-813.

KROYER, H. 1843. Beskrivelse af nogle arter og slaegter af Caprellina: med indledende bemaerkninger om Laemodipoda og deres plads i systemet. Naturhist. Tidsskr. 4:490-518, 585-616.

LAUBITZ, D.R. 1993. Caprellidea (Crustacea: Amphipoda): towards a new synthesis. J. Nat. Hist. 27:965-976. http://dx.doi org/10.1080/00222939300770591

LAUBITZ, D.R. 1995. Caprellidea (Crustacea: Amphipoda) from the Southern and Western Indian Ocean. Mésogée. 54:81-100.

LEITE, F.P.P., TANAKA, M.O., SUDATTI, D.B. \& GEBARA, R.S. 2007. Diel density variation of amphipods associated with Sargassum beds from two shores of Ubatuba, Southeastern, Brazil. Iheringia Ser. Zool. 97(4):400-405. http://dx.doi.org/10.1590/S0073-47212007000400007

MASUNARI, S. \& TAKEUCHI, I. 2006. Redescription of Caprella dilatata Kroyer, 1843 (Caprellidae: Amphipoda: Crustacea) from Brazil, with note on its biogeographical distribution in South America. Zootaxa. 1298:49-60.

McCAIN, J.C. 1968. The Caprellidae (Crustacea: Amphipoda) of the Western North Atlantic. U.S. Nat. Mus. Bull. 278:1-147. http://dx.doi.org/10.5479/ si.03629236.278

MCCAIN, J.C. \&. STEINBERG, J.E. 1970. Amphipoda-I, Caprellidea- I. Crustaceorum Catalogus 2:1-78.

MITTMANN, J. \& MÜLLER, Y.M.R. 1998. Contribuição ao conhecimento da biologia de Caprella penantis (Leach) (Crustacea, Amphipoda) da Ilha de Anhatomirim, Santa Catarina. Rev. Bras. Zool. 15(1):95-100.

MYERS, A.A. \& LOWRY, J.K. 2003. A phylogeny and a new classification of the Corophiidea Leach, 1814 (Amphipoda). J. Crust. Biol. 23(2):443485. http://dx.doi.org/10.1651/0278-0372(2003)023[0443:APAANC] 2.0.CO;2

NEVES, C.S. 2006. Bioinvasão mediada por embarcações de recreio na Baía de Paranaguá, PR e suas implicações para a Conservação. Dissertação de Mestrado, Universidade Federal do Paraná, Paraná.

PINCZON DU SEL, G., BLANC, A. \& DAGUZAN, J. 2000. The diet of the cuttlefish Sepia officinalis L. (Mollusca: Cephalopoda) during its life cycle in the Northern Bay of Biscay (France). Aquat. Sci. 61:167-178. http://dx.doi.org/10.1007/PL00001329

QUITETE, J.M.P.A. 1971. Fallotritella montoucheti, nova espécie de Caprellidae da costa brasileira (Crustacea: Amphipoda). Atas Soc. Biol. 14:189-192.

SAY, T. 1818. An account of the Crustacea of the United States. J. Acad. Nat. Sei. Philadelphia 1:57-441.

SEREJO, C.S. 1998. Gammaridean and Caprellidean fauna (Crustacea) associated with the sponge Dysidea fragilis Johnston at Arraial do Cabo, Rio de Janeiro, Brazil. B. Mar. Sci. 63(2):363-385.

TARARAM, S., WAKABARA, Y. \& LEITE, F.P.P. 1986. Vertical distribution of amphipods living on algae of a Brazilian intertidal rocky shore. Crustaceana 51(2):183-187. http://dx.doi.org/10.1163/156854086X00665

THIEL, M., GUERRA-GARCÍA, J.M., LANCELOTTI, D.A. \& VASQUEZ, N. 2003. The distribution of littoral caprellids (Crustacea: Amphipoda: Caprellidea) along the Pacific coast of continental Chile. Rev. Chil. Hist. Nat. 76:297-312. http://dx.doi.org/10.4067/S0716-078X2003000200014

VOLBEHR, U. \& RACHOR, E. 1997. The association between the caprellid Pariambus typicus Krøyer (Crustacea, Amphipoda) and ophiuroids. Hydrobiologia 355:71-76. http://dx.doi.org/10.1023/A:1003002817720 


\section{Chave de identificação para as espécies ocorrentes no litoral do Paraná e de Santa Catarina}

1a. Pereópodos 3 e 4 presentes

1b. Pereópodos 3 e 4 ausentes

2a. Pereópodos 3 e 4 com seis segmentos (Figura 1a)

Phtisica marina

2b. Pereópodos 3 e 4 com um (Figura 1b) ou dois segmentos

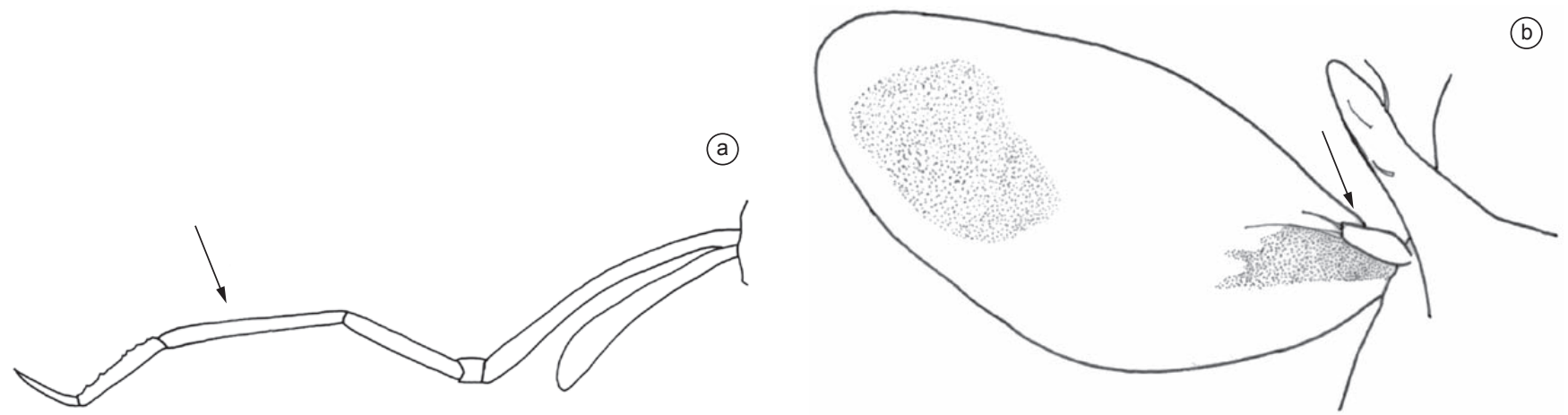

Figura 1

Figure 1

3a. Pereópodos 5 com três segmentos (Figura 2a). Mayerella sp.
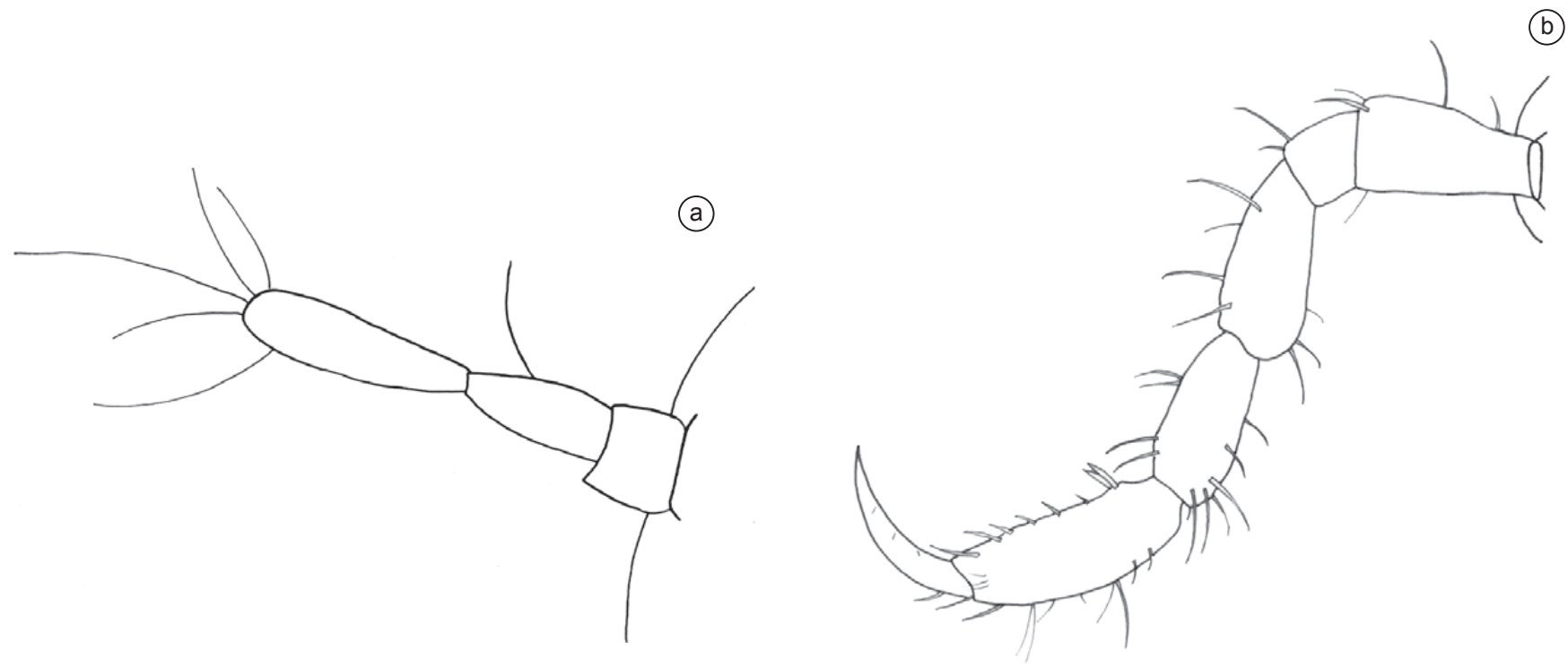

Figura 2

Figure 2

4a. Palpo mandibular representado por uma cerda única (Figura 3a), projeção triangular presente no pereonito 2 nos machos (Figura 10a) .. ..Paracaprella pusilla

4b. Palpo mandibular com três segmentos, segundo segmento com uma cerda simples e terceiro com uma protuberância e fórmula setal $1-4-1$ (Figura 3b), corpo espinhoso em ambos os sexos (Figura 10b) Pseudaeginella montoucheti 
(a)

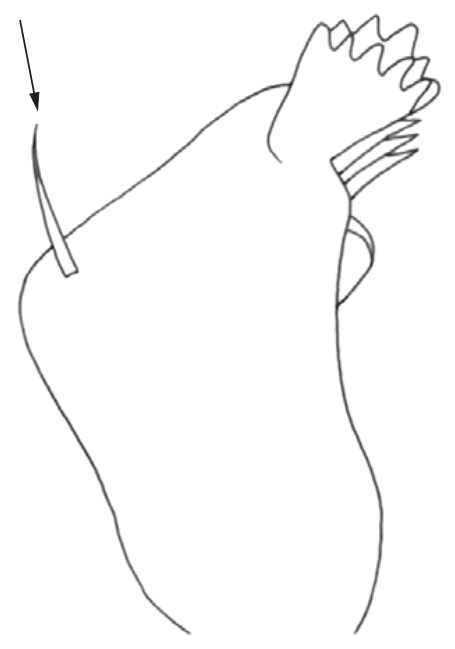

(b)

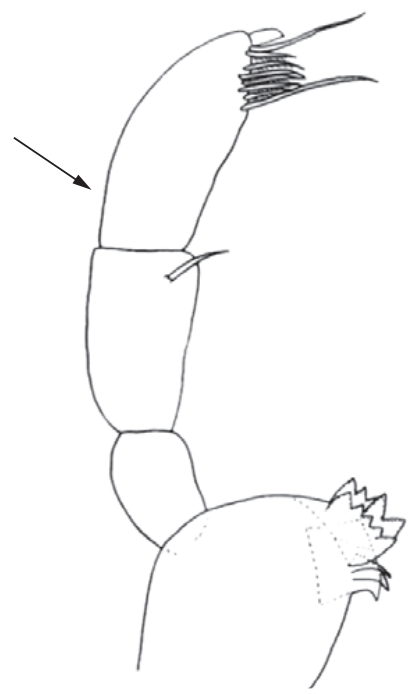

Figura 3

Figure 3

5a. Cabeça com uma projeção ou espinho dirigido anteriormente (Figura 4a) 6

5b. Cabeça sem uma projeção ou espinho dirigido anteriormente (Figura 4b) 8

(a)
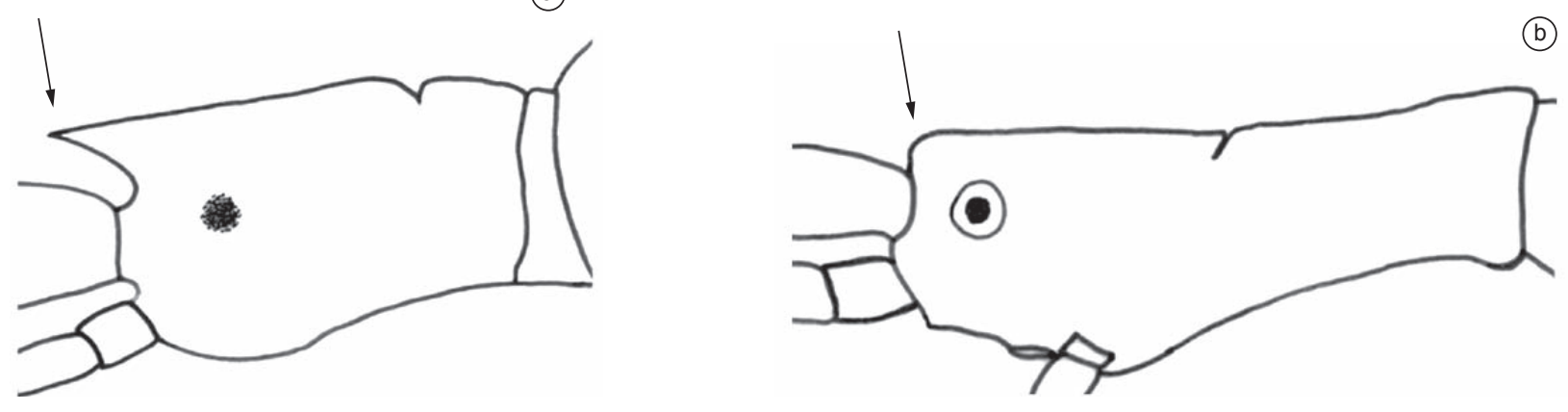

Figura 4

Figure 4

6a. Pereonitos 1 e 2 alongados nos machos, brânquias elípticas em ambos os sexos (Figura 5a). Caprella scaura 6b. Pereonitos 1 e 2 curtos, brânquias grandes e arredondadas em ambos os sexos (Figura 5b) ...... 7

(a)
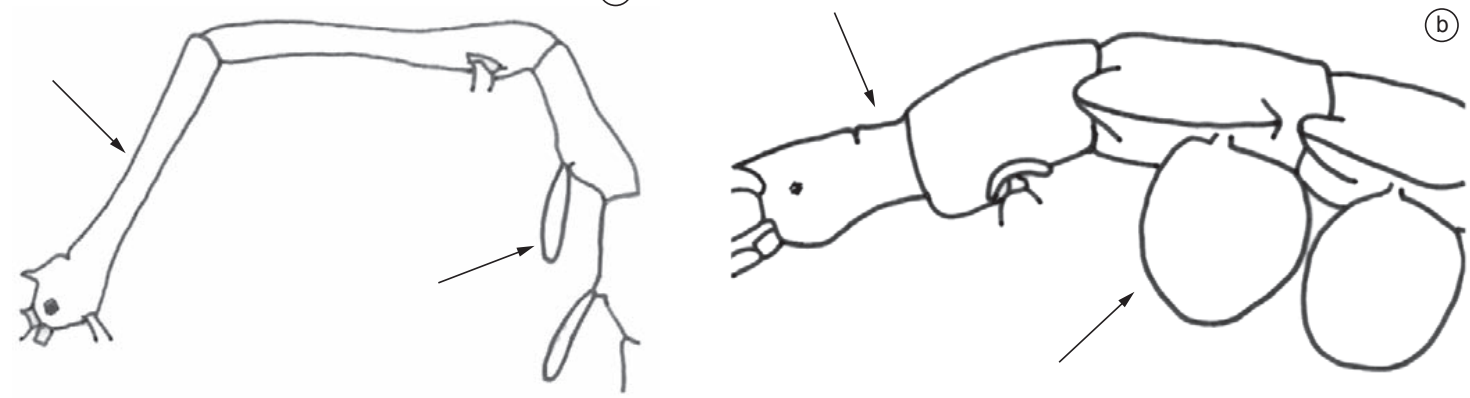

Figura 5

Figure 5 
Lacerda, M.B. \& Masunari, S

7a. Palma do gnatópodo 2 proximalmente dilatada e convexa, um espinho mediano e um processo trapezóide subdistalmente (Figura 6a) ...

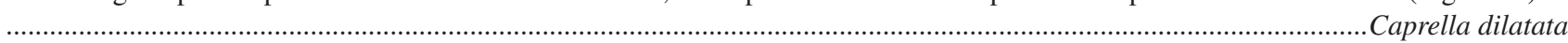

7b. Palma do gnatópodo 2 com um espinho proximal (Figura 6b). Caprella penantis

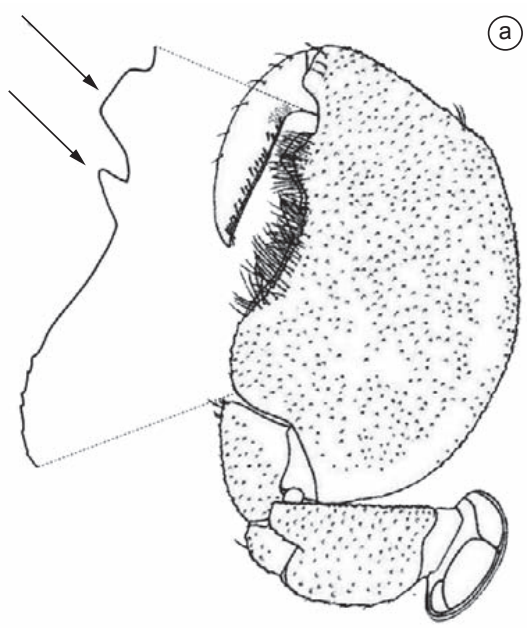

(a)

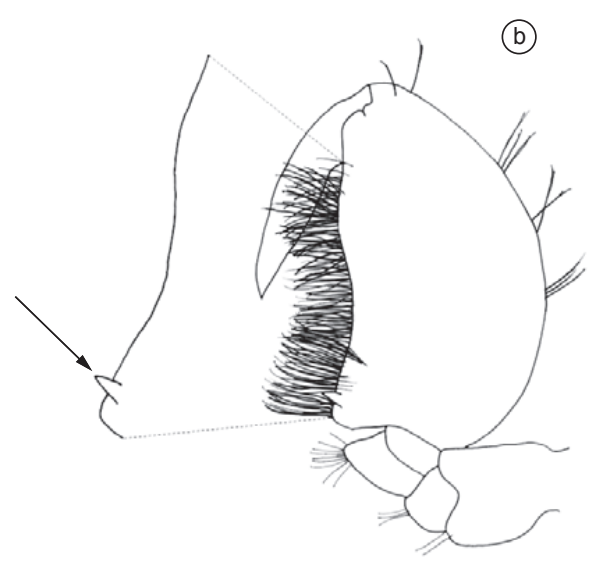

Figura 6. a. Adaptado de Masunari \& Takeuchi (2006); b. Adaptado de McCain (1968).

Figure 6. a. Adapted from Masunari and Takeuchi (2006); b. Adapted from McCain (1968)

8a. Espinho ventral presente entre as inserções dos gnatópodos 2 (Figura 7)

Caprella equilibra

8b. Espinho ventral ausente entre a inserção dos gnatópodos 2

Caprella danilevskii

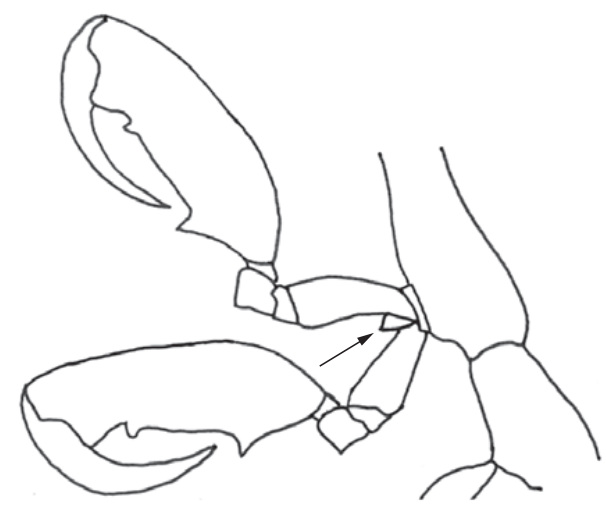

Figura 7. Desenho esquemático dos gnatópodos 2 indicando o espinho ventral presente entre as inserções dos mesmos

Figure 7. Outline of the gnathopods 2 indicating the ventral spine present between their insertion. 